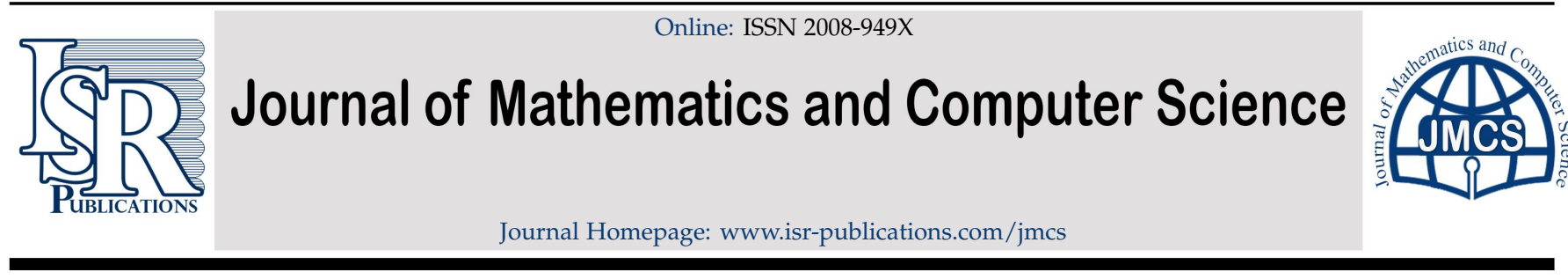

\title{
Wavelet neural network based controller design for non- affine nonlinear systems
}

\author{
Pramendra Kumar*, Vikas Panwar \\ Department of Applied Mathematics, Gautam Buddha University, India.
}

\begin{abstract}
This paper addresses the design of wavelet neural network(WNN) based control scheme for non-affine nonlinear system with unknown control direction. Wavelet neural network is employed to approximate the uncertain part of control system. Since the learning capability of WNN is superior than any conventional NN for system identification. The update laws are derived from Lyapunov stability theory with Nussbaum technique so that all signals in closed loop system are stable and bounded. Finally, simulation example and analysis are provided to prove the effectiveness of controller.
\end{abstract}

Keywords: Non-affine Nonlinear system, Lyapunov stability theory, wavelet neural network, Nussbaum function.

2020 MSC: 34D08, 93D05.

(C)2022 All rights reserved.

\section{Introduction}

The control of systems with uncertain non-affine nonlinear dynamics has been a predominant focused area for decades. For the systems with uncertainties that can be parametrized linearly, the variety of adaptive controllers [8, 23, 24] have been utilized in the past. Some recent adaptive neural, fuzzy and wavelet controllers have also been reported in the literature, where the systems are not linear in the parameters $[2,6,14,19,27]$. Several learning schemes have also been studied for the systems where the disturbance is periodic [1]. Many methods have been used to compensate the structured uncertainty, unstructured uncertainty and unknown disturbance with in the system like sliding mode controllers [24, 25], robust controller [21], neural network and fuzzy controllers [10, 20].

In [7] a class of SISO nonlinear control systems has been considered in which the unknown function depends on the response and its derivatives. In practice, there are numerous systems which can be represented by this kind of control systems. In the paper [27], the authors developed a neural network based robust adaptive scheme for a class of nonlinear systems represented by input-output models with un-modeled dynamics. In [6], the wavelet adaptive backstepping control (WABC) system is presented for a second order class of nonlinear systems. The WABC consists of a neural backstepping controller and a robust controller. Even further, entering in the analysis field, neural networks are also trained to estimate

\footnotetext{
*Corresponding author

Email addresses: kumar .pramendra@rediffmail.com (Pramendra Kumar), vikasdma@gmail.com (Vikas Panwar)

doi: $10.22436 /$ jmcs.024.01.05
}

Received: 2020-09-05 Revised: 2020-09-26 Accepted: 2020-11-14 
properties of complex theoretical problems formulated by partial differential equations (related, for instance, to porous media or membrane structures); [11, 12, 26] moreover, the Galerkin method, employed to approximate solutions to general PDEs, uses a deep neural network instead of a linear combination of basis functions.

In [13] a robust adaptive fuzzy controller is presented for non-affine nonlinear system represented by input-output models. The Fuzzy logic and Nussbaum theory are utilized to fix the structured uncertainty of given system. In the work of [9], an RBF neural adaptive control scheme is proposed for a class of uncertain non-affine nonlinear system. In the paper [17], the authors presented a novel tracking controller for a class of uncertain non-affine systems with time-varying asymmetric output constraints. The stability of non-affine nonlinear system is much more challenging than that of linear one. In spite of, several control systems fall into such category, where the structure is non-affine nonlinear like chemical system, wind turbine, aircraft flight control and mechanical systems [18, 28] etc.

In this paper, we have proposed a wavelet neural network based controller for a class of non-affine nonlinear systems. First, the unconstrained non-affine nonlinear system is transformed into constrained normal form system. Then converted non-affine system transformed into affine one using some mathematical expansion method. The WNN is utilized to approximate the unknown affine part of the control system. The update laws of WNN are derived via Lyapunov synthesis and hence all the the signals in closed-loop system are proved stable along with the control law. Finally the theoretical results are given through numerical simulation studies.

The rest of the paper is as follows. Section 2 gives the problem formulation, description of wavelet neural network and Nussbaum function. In Section 3 the control law and stability analysis are presented. An example is presented in Section 4 to demonstrate the effectiveness of addressed controller. Finally, the conclusion of this paper is described in the Section 5.

\section{Problem formulation}

Lets us consider the following single input-single output (SISO) non-affine nonlinear system given by

$$
y^{n}=f(P, Q) \text {, }
$$

where $P=\left[y, \dot{y}, \ddot{y}, \ldots, y^{n-1}\right]$ and $Q=\left[u, \dot{u}, \ddot{u}, \ldots, u^{m}\right] ; u, y \in R$ are the input and output of system (2.2); $\dot{y}, \ddot{y}, \ldots$ are the first and higher order derivative of $y ; \dot{u}, \ddot{u}, \ldots$ are the first and higher order derivative of $u$; and $f$ is the smooth unknown nonlinear function. Let

$$
x_{1}=y, x_{2}=\dot{y}, \ldots, x_{n}=y^{(n-1)}, \quad z_{1}=u, z_{2}=\dot{u}, \ldots, z_{m}=u^{(m-1)} .
$$

Using the above substitution, the system (2.1) can be written as

$$
\left\{\begin{array}{l}
\dot{x}_{i}=x_{i+1},(i=1,2,3, \ldots, n-1), \\
\dot{x}_{n}=f(x, z, v), \\
\dot{z}_{j}=z_{j+1},(j=1,2,3, \ldots, m-1) \\
\dot{z}_{m}=v
\end{array}\right.
$$

where $x=\left[x_{1}, x_{2}, \ldots, x_{n}\right]^{\top}, z=\left[z_{1}, z_{2}, \ldots, z_{m}\right]^{\top}$ are the state variables and available for system (2.2), and $v=u^{m}$ is the control input of augmented system (2.2). The initial states of the system are chosen such that $z(0) \in Z_{0}, x(0) \in X_{0}$, where $Z_{0} \& X_{0}$ are the compact subsets of $R^{m} \& R^{n}$, respectively. Using mean value theorem, the function $f$ in system (2.2) can be written as:

$$
f(x, z, v)=f\left(x, z, v^{*}\right)+g\left(v-v^{*}\right),
$$

where $g=\frac{\partial f(x, z, v)}{\partial v}$ and $v^{*} \in V_{0} \subset R$. By choosing $v^{*}=0,[5,22]$, the function $f(x, z, v)$ in Eq. (2.3) can 
be expressed as:

$$
f(x, z, v)=f(x, z)+g v
$$

Using the above equation, the system (2.2) can be transformed into the form:

$$
\left\{\begin{array}{l}
\dot{x}_{i}=x_{i+1}, \\
\dot{x}_{n}=f(x, z)+g v, \\
\dot{z}_{j}=z_{j+1} \\
\dot{z}_{m}=v
\end{array}\right.
$$

Let $Y_{d}=\left[y_{d}, y_{d}^{(1)}, y_{d}^{(2)}, \ldots, y_{d}^{(n-1)}\right]$. Regarding the development of control law, we will make the following assumptions.

Assumption 1: The desired signal $y_{d}(t)$ and its derivatives $y_{d}^{(n)}(t)$ are smooth and bounded.

Assumption 2: The sign of nonlinear function $g=\frac{\partial f}{\partial \nu}$ is unknown and satisfies $0<g_{\min } \leqslant|g| \leqslant g_{\max }$ where $g_{\min }$ and $g_{\max }$ are the positive constants.

Assumption 3: The disturbance term $\epsilon^{*}$ is bounded and it is bounded by $\epsilon>0$ such that $\left|\epsilon^{*}\right| \leqslant \epsilon$.

Let $e=\left[e_{1}, e_{2}, \ldots, e_{n}\right]^{\top}$, where $e_{1}=x_{1}-y_{d}, e_{2}=x_{2}-y_{d}^{(1)}, \ldots, e_{n}=x_{n}-y_{d}^{(n-1)}$. Define a new variable $e_{s}$ (sliding surface) as follows:

$$
e_{s}=\left(\frac{d}{d t}+\alpha\right)^{n-1} e_{1}=[\Lambda 1] e
$$

where $\Lambda=\left[\alpha^{n-1},(n-1) \alpha^{n-2}, \frac{(n-1)(n-2)}{2 !} \alpha^{n-3}, \ldots,(n-1) \alpha\right]^{\top}$ and $\alpha$ is a positive constant. It has been shown in [24] that $e_{s} \longrightarrow 0$ as $t \longrightarrow \infty$. From which, we can conclude that $e$ and consequently all the derivatives of e up to $(n-1)^{\text {th }}$ order converge to zero. Using (2.4), the time derivative of (2.5) can be written as

$$
\dot{e}_{s}=f(s)+\beta+g v-y_{d}^{(n)},
$$

where $s=\left[x^{\top}, z^{\top}\right]^{\top}$ and $\beta=\left[\begin{array}{ll}0 & \Lambda\end{array}\right]^{\top}$ e. Before the construction of controller design, we first introduce the wavelet neural network to approximate the unknown function $f(s)$ in (2.6) and basic of Nussbaum function.

\subsection{Wavelet neural network}

Mathematically, Wavelet neural network is a nonlinear mapping from an input space $R^{p}$ to an output space $R(p=m+n)$, i.e., $f(s): R^{p} \rightarrow R$ defined as [19]:

$$
f(s)=w^{\top} \Psi(s, d, c),
$$

where $s=\left[s_{1}, s_{2}, \ldots, s_{p}\right]^{\top} \in R^{p}$ is the input vector, $w=\left[w_{1}, w_{2}, \ldots, w_{q}\right]^{\top} \in R^{q}$ is the weight vector of the output layer, $q$ is the number of neurons in the translation layers, $d=\left[d_{1}^{\top}, d_{2}^{\top}, \ldots, d_{q}^{\top}\right]^{\top} \in R^{q p}$ is dilation parameters vector, $c=\left[c_{1}^{\top}, c_{2}^{\top}, \ldots, c_{q}^{\top}\right]^{\top} \in R^{q p}$ is the translation parameters vector, $d_{i}=$ $\left[d_{i 1}, d_{i 2}, \ldots, d_{i p}\right] \in R^{p}, c_{i}=\left[c_{i 1}, c_{i 2}, \ldots, c_{i p}\right] \in R^{p}$, where $i=1,2, \ldots, q$ and $\Psi(., .)=.\left[\psi_{1}, \psi_{2}, \ldots, \psi_{q}\right]^{\top} \in$ $\mathrm{R}^{\mathrm{q}}$ is the translation layer output, given as:

$$
\psi_{i}=\omega_{i}(s) \exp \left(-\frac{\sum_{j=1}^{p} d_{i j}^{2}\left(s_{j}-c_{i j}\right)^{2}}{2}\right),
$$


where $\omega_{i}(s)=\prod_{j=1}^{p}\left(1-d_{i j}^{2} s_{j}^{2}\right)$ is the "Mexican hat" mother wavelet function. According to universal approximation theorem [29], $\exists$ a wavelet neural network such that

$$
f(s)=f^{*}(s)+\delta^{*}(s)=w^{* T} \Psi\left(s, d^{*}, c^{*}\right)+\delta^{*}(s),
$$

where $\delta^{*}(s)$ is the bounded wavelet neural network approximation error $\left|\delta^{*}(s)\right| \leqslant \delta_{m} ; \delta_{m}>0 . w^{*}, d^{*}$, and $c^{*}$ are the bounded optimal parameter values of $w, d$, and $c$. Though, it is not easy to find the exact values of above mentioned optimal parameters, so we use estimated values of the optimal parameters provided by:

$$
\hat{f}(s)=\hat{w}^{\top} \Psi(s, \hat{d}, \hat{c}), \quad \hat{f}(s)=\hat{w}^{\top} \hat{\Psi},
$$

where $\hat{w}, \hat{\mathrm{d}}$ and $\hat{c}$ are the estimated values of $w, d$, and $c$. Assuming $\tilde{w}=w^{*}-\hat{w}, \tilde{d}=d^{*}-\hat{d}, \tilde{c}=c^{*}-\hat{c}, \tilde{\Psi}=$ $\Psi^{*}-\hat{\Psi}=\left[\tilde{\psi}_{1}, \tilde{\psi}_{2}, \ldots, \tilde{\psi}_{\mathrm{q}}\right]^{\top}$. Now, using the above notations, we can define:

$$
\begin{aligned}
& \tilde{f}=f-\hat{f}=f^{*}-\hat{f}+\delta^{*}, \\
& \tilde{f}=w^{*} \Psi^{*}-\hat{w}^{\top} \hat{\Psi}+\delta^{*}=\left(\hat{w}^{\top}+\tilde{w}^{\top}\right)(\hat{\Psi}+\tilde{\Psi})-\hat{w}^{\top} \hat{\Psi}+\delta^{*}, \\
& \tilde{f}=\tilde{w}^{\top} \hat{\Psi}+\hat{w}^{\top} \tilde{\Psi}+\tilde{w}^{\top} \tilde{\Psi}+\delta^{*} .
\end{aligned}
$$

Using the Taylor expansion method, the semi linear form of $\tilde{\psi}$ can be obtained for the derivation of online update laws of wavelet neural network parameters:

$$
\begin{aligned}
& \tilde{\psi}_{i}=\sum_{j=1}^{p}\left(\frac{\partial \psi_{i}}{\partial d_{i j}}\right)_{\left(\hat{d}_{i j}, \hat{c}_{i j}\right)} \tilde{d}_{i j}+\left(\frac{\partial \psi_{i}}{\partial c_{i j}}\right)_{\left(\hat{d}_{i j}, \hat{c}_{i j}\right)} \tilde{c}_{i j}+\eta_{i} ; i=1,2, \ldots, q, \\
& \tilde{\psi}_{i}=A_{i} \tilde{d}_{i}+B_{i} \tilde{c}_{i}+\eta_{i},
\end{aligned}
$$

where $A_{i}=\left[\frac{\partial \psi_{i}}{\partial d_{i 1}}, \frac{\partial \psi_{i}}{\partial d_{i 2}}, \ldots, \frac{\partial \psi_{i}}{\partial d_{i p}}\right], B_{i}=\left[\frac{\partial \psi_{i}}{\partial c_{i 1}}, \frac{\partial \psi_{i}}{\partial c_{i 2}}, \ldots, \frac{\partial \psi_{i}}{\partial c_{i p}}\right]$, and $\eta_{i}$ represent the higher order terms obtained in the expansion of Taylor series. Equation (2.8) can be written in the vector form as:

$$
\tilde{\Psi}=A \tilde{d}+B \tilde{c}+\eta,
$$

where $A=\operatorname{blokdiag}\left[A_{1}, A_{2}, \ldots, A_{q}\right] \in R^{q \times q p}, B=\operatorname{blokdiag}\left[B_{1}, B_{2}, \ldots, B_{q}\right] \in R^{q \times q p}$, and $\eta=\left[\eta_{1}, \eta_{2}, \ldots, \eta_{q}\right] \in$ $\mathrm{R}^{\mathrm{q}}$. Using Eq. (2.9) in (2.7), we get the following form of $\tilde{f}$ :

$$
\begin{aligned}
& \tilde{f}=\tilde{w}^{\top} \hat{\Psi}+\hat{w}^{\top}(A \tilde{d}+B \tilde{c}+\eta)+\tilde{w}^{\top}(A \tilde{d}+B \tilde{c}+\eta)+\delta^{*}, \\
& \tilde{f}=\tilde{w}^{\top} \hat{\Psi}+\hat{w}^{\top} A \tilde{d}+\hat{w}^{\top} B \tilde{c}+\hat{w}^{\top} \eta+\tilde{w}^{\top} A\left(d^{*}-\hat{d}\right)+\tilde{w}^{\top} B\left(c^{*}-\hat{c}\right)+\tilde{w}^{\top} \eta+\delta^{*}, \\
& \tilde{f}=\tilde{w}^{\top}(\hat{\Psi}-A \hat{d}-B \hat{c})+\hat{w}^{\top} A \tilde{d}+\hat{w}^{\top} B \tilde{c}+\epsilon^{*},
\end{aligned}
$$

where $\epsilon^{*}=\hat{w}^{\top} \eta+\tilde{w}^{\top} A d^{*}+\tilde{w}^{\top} B c^{*}+\tilde{w}^{\top} \eta+\delta^{*}$ is the disturbance term, which represents the estimation errors. By using the above wavelet neural network approximation of function $f(s)$, the error dynamics of system (2.2) can be written as:

$$
\dot{e}_{s}=\hat{w}^{\top} \hat{\Psi}+\tilde{w}^{\top}(\hat{\Psi}-A \hat{d}-B \hat{c})+\hat{w}^{\top} A \tilde{d}+\hat{w}^{\top} B \tilde{c}+\beta+g v-y_{d}^{(n)}+\epsilon^{*} .
$$

\subsection{Nussbaum function}

A function is said to be a Nussbaum function, if it satisfies the following two conditions $[4,16]$

$$
\left\{\begin{array}{l}
\lim _{\mathrm{r} \longrightarrow+\infty} \sup \frac{1}{\mathrm{r}} \int_{0}^{\mathrm{r}} \mathrm{N}(\zeta) \mathrm{d} \zeta=+\infty, \\
\lim _{\mathrm{r} \longrightarrow+\infty} \inf \frac{1}{\mathrm{r}} \int_{0}^{\mathrm{r}} \mathrm{N}(\zeta) \mathrm{d} \zeta=-\infty .
\end{array}\right.
$$

There are many functions that satisfy the above two conditions. The continuous functions $e^{t^{2}} \sin t, t^{2} \cos t$, $e^{t^{2}} \cos \left(\frac{\pi}{2} t\right)$ satisfy the Eq. (2.11) and hence they are the good examples of Nussbaum functions. The following lemma is used to design the controller of the system defined in Eq. (2.2). 
Lemma $2.1([3,15])$. Let $\mathrm{V}(\mathrm{t})$ and $\zeta(\mathrm{t})$ be the smooth functions defined on $\left[0, \mathrm{t}_{\mathrm{f}}\right)$ with $\mathrm{V}(\mathrm{t}) \geqslant 0, \forall \mathrm{t} \in\left[0, \mathrm{t}_{\mathrm{f}}\right)$ and $\mathrm{N}(\zeta)$ be an Nussbaum function. If the following inequality holds:

$$
0 \leqslant \mathrm{~V}(\mathrm{t}) \leqslant \mathrm{a}_{0}+\int_{0}^{\mathrm{t}}(\mathrm{H}(\cdot) \mathrm{N}(\zeta)+1) \dot{\zeta} \mathrm{d} \sigma,
$$

where $\mathrm{H}(\cdot)$ is a piecewise time varying continuous function that belongs to the unknown closed intervals $\mathrm{I}=\left[\mathrm{t}^{-}, \mathrm{t}^{+}\right]$ with $0 \notin \mathrm{I}$ and $\mathrm{a}_{0}$ is a constant, then $\mathrm{V}(\mathrm{t}), \zeta(\mathrm{t})$ and $\int_{0}^{\mathrm{t}} \mathrm{H}(\cdot) \mathrm{N}(\zeta) \dot{\zeta} \mathrm{d} \sigma$ are bounded on $\left[0, \mathrm{t}_{\mathrm{f}}\right)$.

\section{Controller design and stability analysis}

In this section, the controller design, stability analysis and update laws are presented for the given system (2.2).

Theorem 3.1. Consider the error dynamics represented by (2.10). Let, the control law be chosen as:

$$
\begin{aligned}
& v=\mathrm{N}(\zeta) \dot{\zeta}, \\
& \dot{\zeta}=e_{\mathrm{s}} \hat{\mathcal{W}}^{\mathrm{T}} \hat{\Psi}+k e_{\mathrm{s}}^{2}+e_{\mathrm{s}} \beta-e_{\mathrm{s}} \mathrm{y}_{\mathrm{d}}^{(\mathrm{n})},
\end{aligned}
$$

with $\mathrm{k}$ is a positive constant. If the WNN parameters update laws are chosen as:

$$
\begin{aligned}
\dot{\hat{w}} & =\Gamma_{w}\left((\hat{\Psi}-A \hat{d}-B \hat{c}) e_{s}-\gamma \hat{w}\right), \\
\hat{d} & =\Gamma_{d} A^{\top} \hat{w} e_{s}, \\
\hat{c} & =\Gamma_{c} B^{\top} \hat{w} e_{s},
\end{aligned}
$$

let the assumptions hold from 1-3. Consider a compact set $\Omega_{(x, z)} \in \mathrm{R}^{\mathrm{m}+\mathrm{n}}$, for any $(x(0), z(0)) \in \Omega_{(x, z)}, \exists$ the positive constants $\mathrm{k}$ and $\gamma$ such that all the signals in closed loop system are bounded and the tracking error $\mathrm{e}(\mathrm{t}) \rightarrow 0$, where the matrices $\Gamma_{w} \in \mathrm{R}^{\mathrm{q} \times \mathrm{q}}, \Gamma_{\mathrm{d}} \in \mathrm{R}^{\mathrm{qp} \times \mathrm{qp}}$ and $\Gamma_{\mathrm{c}} \in \mathrm{R}^{\mathrm{qp} \times \mathrm{qp}}$ are positive definite.

Proof. Let us take the following as Lyapunov function:

$$
\mathrm{V}(\mathrm{t})=\frac{1}{2} e_{\mathrm{s}}^{2}+\frac{1}{2} \tilde{w}^{\top} \Gamma_{w}^{-1} \tilde{w}+\frac{1}{2} \tilde{\mathrm{d}}^{\top} \Gamma_{\mathrm{d}}^{-1} \tilde{\mathrm{d}}+\frac{1}{2} \tilde{\mathrm{c}}^{\mathrm{T}} \Gamma_{\mathrm{c}}^{-1} \tilde{\mathrm{c}}
$$

Using the fact that $\dot{\tilde{w}}=-\dot{\hat{w}}, \dot{\tilde{\mathrm{d}}}=-\dot{\hat{\mathrm{d}}}, \dot{\tilde{\mathrm{c}}}=-\dot{\hat{\mathrm{c}}}$, the derivative of $\mathrm{V}$ with respect to time can be written as:

$$
\dot{V}(t)=e_{s} \dot{e_{s}}-\tilde{w}^{\top} \Gamma_{w}^{-1} \dot{\hat{w}}-\tilde{\mathrm{d}}^{\top} \Gamma_{\mathrm{d}}^{-1} \dot{\hat{\mathrm{d}}}-\tilde{\mathrm{c}}^{\top} \Gamma_{\mathrm{c}}^{-1} \dot{\hat{\mathrm{c}}}
$$

Using (2.10), the Eq. (3.6) can be written as:

$$
\begin{aligned}
\dot{V}(t)= & e_{s}\left(\hat{w}^{\top} \hat{\Psi}+\tilde{w}^{\top}(\hat{\Psi}-A \hat{d}-B \hat{c})+\hat{w}^{\top} A \tilde{d}+\hat{w}^{\top} B \tilde{c}+\beta+g v-y_{d}^{(n)}+\epsilon^{*}\right) \\
& -\tilde{w}^{\top} \Gamma_{w}^{-1} \dot{\hat{w}}-\tilde{d}^{\top} \Gamma_{d}^{-1} \dot{\hat{d}}-\tilde{c}^{\top} \Gamma_{c}^{-1} \dot{\hat{c}} .
\end{aligned}
$$

Substituting the WNN update laws (3.3), (3.4), and (3.5) in Eq. (3.7), yields:

$$
\dot{V}(t)=e_{s} \hat{w}^{\top} \hat{\Psi}+\gamma \tilde{w}^{\top} \hat{w}+e_{s} \beta+e_{s} g v+e_{s} \epsilon^{*}-e_{s} y_{d}^{n},
$$

according assumption $3,\left|\epsilon^{*}\right| \leqslant \epsilon, \epsilon>0$ and using the inequality $e_{s} \epsilon \leqslant \frac{e_{s}^{2}}{4}+\epsilon^{2}$, we can write Eq. (3.8) as:

$$
\dot{V}(t) \leqslant e_{s} \hat{w}^{\top} \hat{\Psi}+\gamma \tilde{w}^{\top} \hat{w}+e_{s} \beta+e_{s} g v+\frac{e_{s}^{2}}{4}+\epsilon^{2}-e_{s} y_{d}^{n}
$$


adding and subtracting $k e_{s}^{2}$ and after substituting (3.1) and (3.2) in Eq. (3.9), yields:

$$
\dot{V}(t) \leqslant-\left(k-\frac{1}{4}\right) e_{s}^{2}+\left(e_{s} g N(\zeta)+1\right) \dot{\zeta}+\gamma \tilde{w}^{\top} \hat{w}+\epsilon^{2} .
$$

Using the inequalities:

$$
\gamma \tilde{w}^{\top} \hat{w}=-\frac{\gamma}{2}\|\tilde{w}\|^{2}-\frac{\gamma}{2}\|\hat{w}\|^{2}+\frac{\gamma}{2}\|\tilde{w}+\hat{w}\|^{2}, \quad \gamma \tilde{w}^{\top} \hat{w} \leqslant-\frac{\gamma}{2}\|\tilde{w}\|^{2}+\frac{\gamma}{2}\left\|w^{*}\right\|^{2},
$$

the Eq. (3.10) can be bounded as:

$$
\dot{V}(\mathrm{t}) \leqslant-\left(\mathrm{k}-\frac{1}{4}\right) e_{s}^{2}-\frac{\gamma}{2}\left(\|\tilde{w}\|^{2}-\left\|w^{*}\right\|^{2}\right)+\epsilon^{2}+\left(e_{s} g N(\zeta)+1\right) \dot{\zeta}
$$

Let us choose $\mathrm{H}=e_{\mathrm{s}} \mathrm{g}$ and $\lambda=\frac{\gamma}{2}\left\|w^{*}\right\|^{2}+\epsilon^{2}$. Then, we have

$$
\dot{\mathrm{V}}(\mathrm{t}) \leqslant-\left(\mathrm{k}-\frac{1}{4}\right) e_{\mathrm{s}}^{2}-\frac{\gamma}{2}\|\tilde{w}\|^{2}+\lambda+(\mathrm{HN}(\zeta)+1) \dot{\zeta}
$$

Assuming that $k_{1}>\frac{1}{4}$, then by integrating Eq. (3.11) on $[0, t]$, yields

$$
\left.0 \leqslant \mathrm{~V}(\mathrm{t}) \leqslant \mathrm{a}_{0}+\int_{0}^{\mathrm{t}}[\mathrm{HN}(\zeta)+1) \dot{\zeta}\right] \mathrm{d} \sigma
$$

where $a_{0}=V(0)+\int_{0}^{\infty} \lambda(t) d t$. By using Lemma 2.1 and Eq. (3.12), we can deduce the boundedness of $\left.\int_{0}^{t}[H N(\zeta)+1) \dot{\zeta}\right] \mathrm{d} \sigma, \mathrm{V}(\mathrm{t})$ and $\dot{\zeta}(\mathrm{t})$ on $\left[0, \mathrm{t}_{\mathrm{f}}\right)$. According to [15], it can be concluded that the system is also true for $t_{f}=\infty$. This proves that all the signals involved in closed loop system (2.2), i.e., $x, z$, and $w$ are bounded. Furthermore, the tracking error is also bounded. This completes the proof.

\section{Simulation results}

For simulation purpose, the following non-affine nonlinear system in normal form is used to validate the effectiveness of proposed controller.

$$
\left\{\begin{array}{l}
\dot{x}_{1}=x_{2} \\
\dot{x}_{2}=x_{3} \\
\dot{x}_{3}=z_{1}+x_{1}-x_{3}+x_{1} x_{2}+x_{2}^{2}+x_{1} x_{3} \sin (v)-z_{1} v+d(t) \\
\dot{z}_{1}=v
\end{array}\right.
$$

where $d(t)=0.1 \sin (15 t)$ is the disturbance term. The control objective is to force the controller that system output $y=x_{1}(t)$ tracks the desired trajectory $y_{d}=0.1 \sin t$. With in this simulation, we have used wavelet neural network to approximate the unknown function $f(x, z)$. Let the input variables of wavelet neural network be $x_{1}, x_{2}, x_{3}$, and $x_{4}=z_{1}$. The wavelet neural network consists of two neurons in the translation layer. The design parameters are selected as: $\beta=6.2, \gamma=0.001, k=0.6$ and the matrices $\Gamma_{w}, \Gamma_{\mathrm{d}}, \Gamma_{\mathrm{c}}$ are selected as $\Gamma_{w}=80 \mathrm{I}_{2}, \Gamma_{\mathrm{d}}=100 \mathrm{I}_{8}$ and $\Gamma_{\mathrm{c}}=100 \mathrm{I}_{8}$. The states are initialized by $[x(0), z(0)]=$ $[.01,0.1,0.1,0.1]$. The initial value of $\zeta=0$. The controller is simulated in MATLAB environment.

To analyze the simulation results of proposed controller, we are taking the following three cases. 
Case 1: In the first case, we are taking the same system based controller by considering that no structured uncertainty and Nussbaum technique are exist, however unknown disturbance is present in the system dynamics.

Case 2: In the second case, we are taking the same system dynamics based controller by considering that no structured uncertainty and unknown disturbance exist in the system.

Case 3: In the third case, the proposed wavelet neural network based controller with all above mentioned constraints in the system is used.

The performance of all three controllers are shown in Figures 1-6 with their error curve. Fig. 1 gives the output of desired trajectory vs system system trajectory of Case 1 controller. Fig. 2 shows the tracking error of Case 1 controller. Fig. 3 shows the system vs desired trajectory of Case 2 controller. Fig. 4 is the tracking error of Case 2 controller. Fig. 5 is the output of the closed loop system vs desired trajectory of Case 3 controller and Fig. 6 is the tracking error of Case 3 controller. From the Figs. 1-6, it is clear that the WNN controller can learn the structured uncertainty effectively. Also, the tracking error of WNN controller is smaller than the other two controllers. The curve of $\zeta$ is shown in Fig. 7.

We are using the scalar valued $L_{2}$ norm for entire error curve to study the performance of controllers from Cases 1-3. The $L_{2}$ norm calculate the RMS average of tracking errors and it is given by:

$$
L_{2}[e]=\sqrt{\frac{1}{t_{f}-t_{0}} \int_{t_{0}}^{t_{f}}\|e(t)\|^{2} d t .}
$$

The smaller $\mathrm{L}_{2}[e]$ norm represents the less tracking error of controller and it reflects the good performance of controller. To check the performance of each Cases 1-3 controllers statistically, the simulation experiment were performed for 20 times with same learning matrices but with different parameters. For each execution, the root mean square values of tracking errors are recorded. The average of these RMS values and variances of Cases 1-3 controllers are plotted in Figs. 8 and 9. An unpaired pooled t-test is used through MS office excel 2013 with significance level $\alpha=0.05$ and equal variances. The pooled t-test is applied for the controller Case 3 with all other Cases 1-2 controllers. The result of $t$-test of root mean square error is shown in Table 1. It can be drawn from the Table 1 that the value of $t$ stat is bigger than the $t$ critical values. Also the $P$ value obtained in one tail and two tail test is smaller than the significance level $\alpha$. Thus it can be concluded that the mean RMS error for the Case 3 controller is less than the other two controllers Cases 1-2 and this difference is statistically significant.

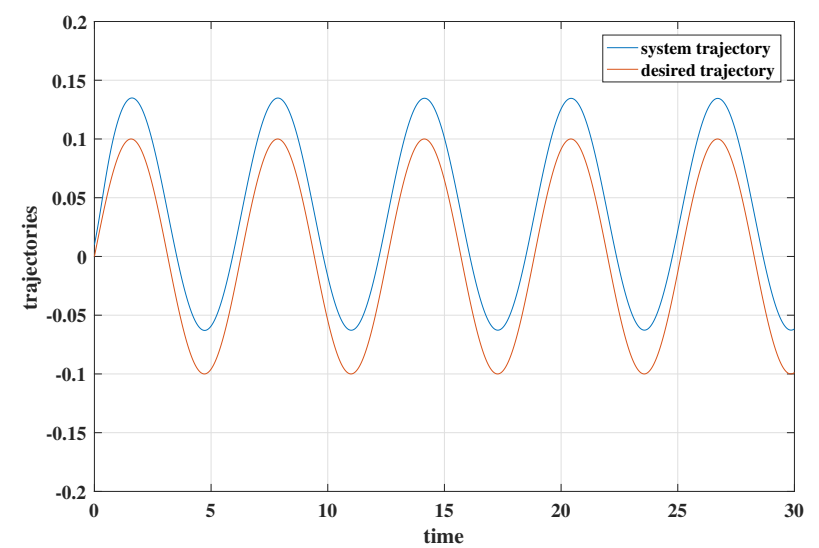

Figure 1: Desired Trajectory vs system trajectory of Case 1 controller.

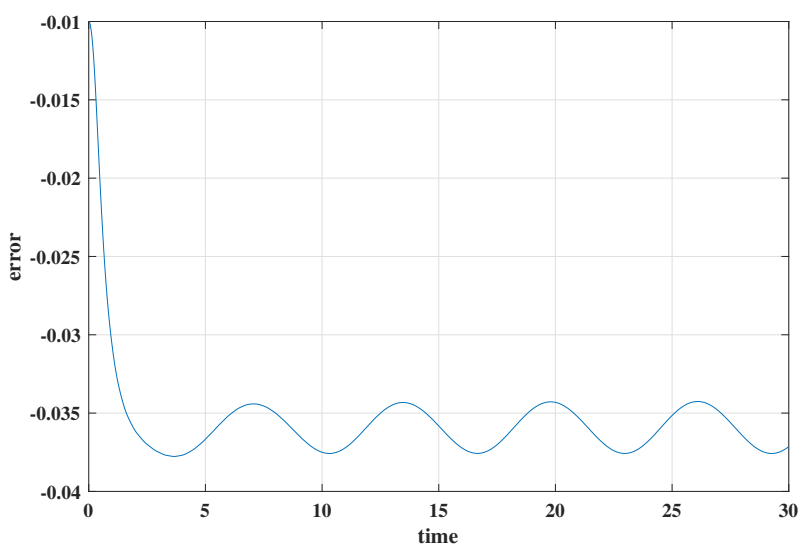

Figure 2: Tracking error of Case 1 controller. 


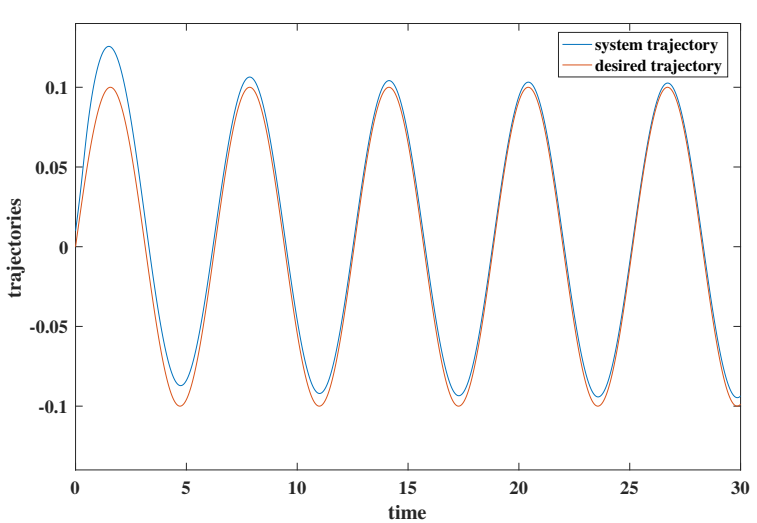

Figure 3: Desired Trajectory vs system trajectory of Case 2 controller.

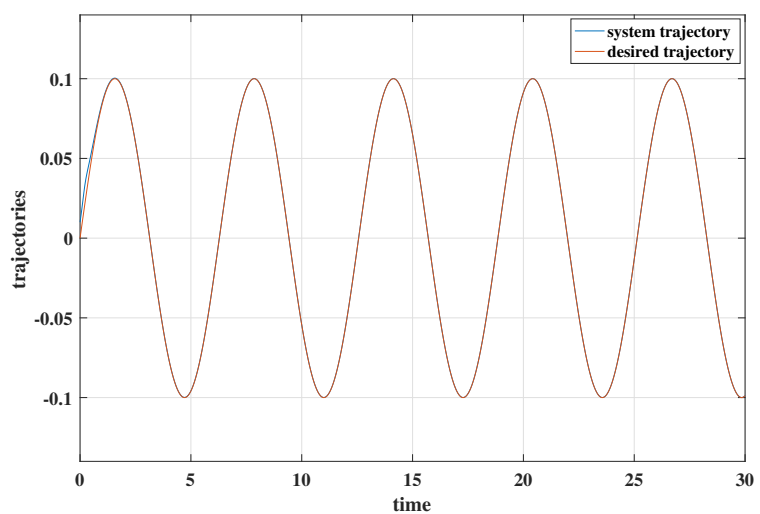

Figure 5: Desired Trajectory vs system trajectory of Case 3 controller.

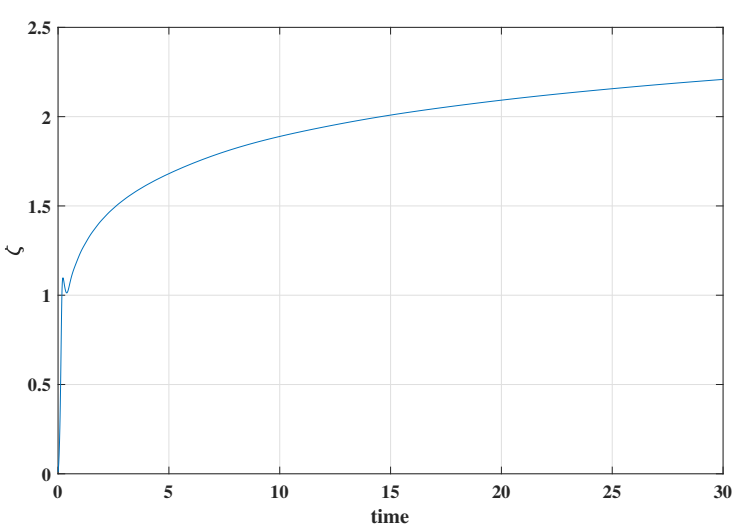

Figure 7: curve of $\zeta$ for Case 3 controller.

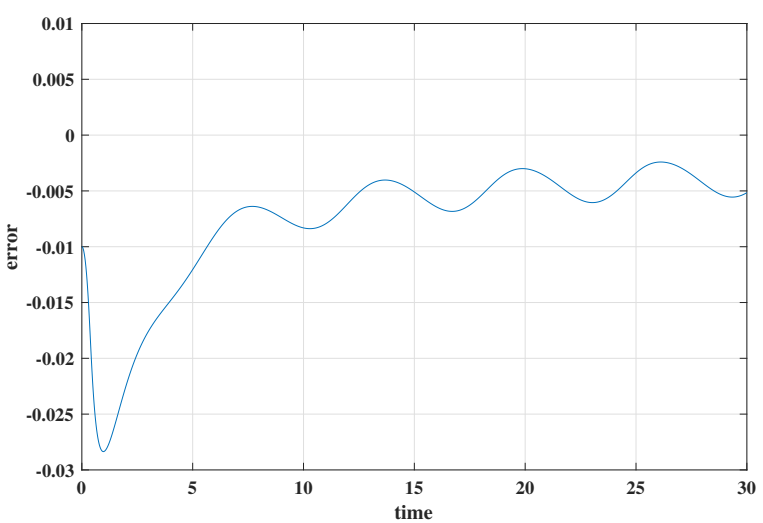

Figure 4: Tracking error of Case 2 controller.

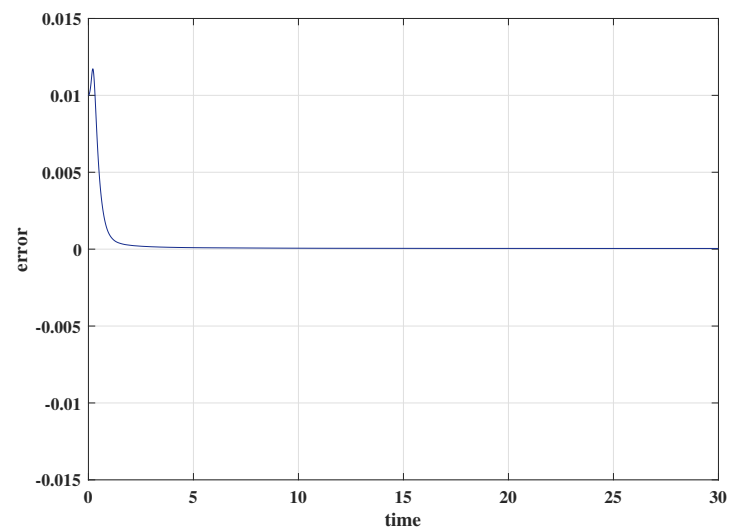

Figure 6: Tracking error of Case 3 controller.

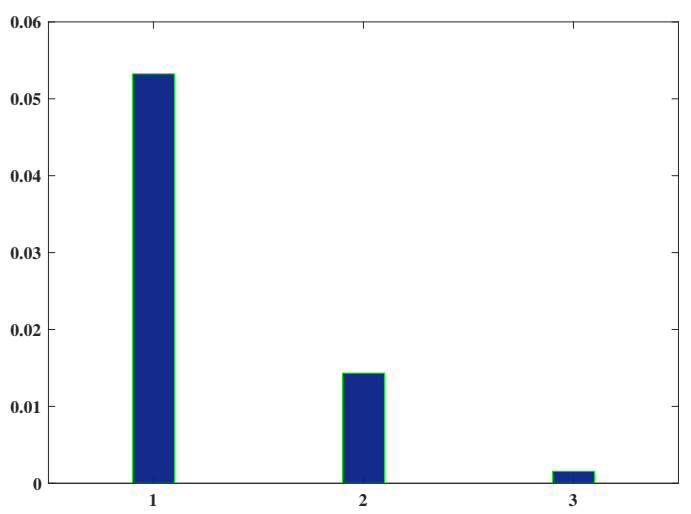

Figure 8: RMS Tracking errors of the controllers 1-3. 


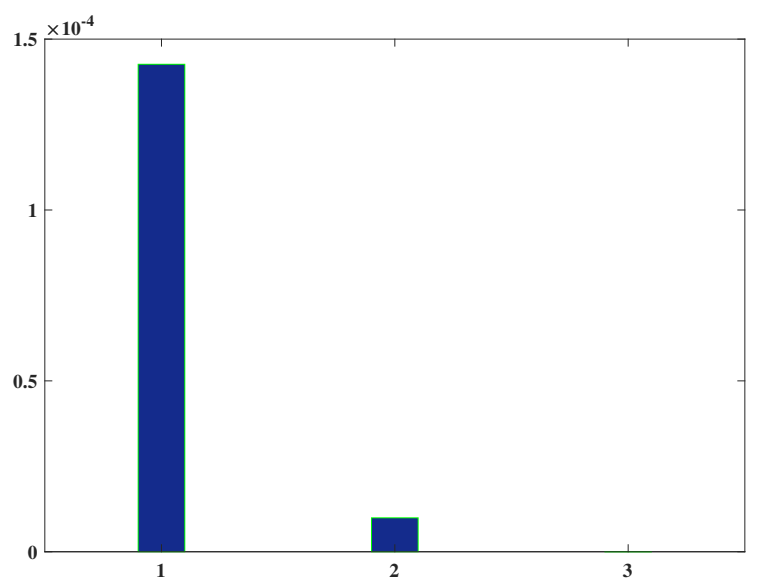

Figure 9: Variance about mean of the controllers 1-3.

Table 1: t-test for two samples assuming equal variances for RMS tracking error.

\begin{tabular}{llll}
\hline & \multicolumn{3}{c}{ RMS tracking error } \\
\cline { 2 - 4 } & Case 1 & Case 2 & Case 3 \\
\hline Mean & $5.32 \times 10^{-2}$ & $1.43 \times 10^{-2}$ & $1.56 \times 10^{-3}$ \\
Variance & $1.42 \times 10^{-4}$ & $9.93 \times 10^{-6}$ & $4.17 \times 10^{-8}$ \\
Observations & 20 & 20 & 20 \\
Pooled variance & $7.13 \times 10^{-5}$ & $4.98 \times 10^{-6}$ & \\
Hypothesized mean difference & 0 & 0 & \\
$\mathrm{df}$ & 38 & 38 & \\
$\mathrm{t}$ stat & 19.35 & 18.08 & \\
$\mathrm{P}(\mathrm{T} \leqslant \mathrm{t})$ one-tail & $1.41 \times 10^{-21}$ & $1.46 \times 10^{-20}$ & \\
$\mathrm{t}$ Critical one-tail & 1.68 & 1.68 & \\
$\mathrm{P}(\mathrm{T} \leqslant \mathrm{t})$ two-tail & $2.82 \times 10^{-21}$ & $2.92 \times 10^{-20}$ & \\
$\mathrm{t}$ Critical two-tail & 2.024 & 2.024 & \\
\hline
\end{tabular}

\section{Conclusion}

In the present work, we have presented an adaptive feedback scheme for a class of SISO non-affine nonlinear systems with unknown controller gain. The WNN neural network and Nussbaum function have been used to fix the unknown dynamics of system (2.2). Using Lyapunov stability theory, it has been proven that the signals in closed loop system are stable and bounded. The computer simulation studies shows that the wavelet neural network controller with updating laws can efficaciously achieve the desired performance. In addition, the unpaired pooled t-test results show that the presented WNN based scheme is shown to be statistically significant in comparison to other controllers.

\section{References}

[1] P. J. Antsaklis, K. M. Passino, Eds., An Introduction to Intelligent and Autonomous Control, Kluwer Academic Publishers, (1993). 1

[2] S. S. Ge, C. C. Hang, T. Zhang, Nonlinear Adaptive Control using neural networks and its applications to CSTR system, J. process. Control, 9 (1999), 313-323. 1

[3] S. S. Ge, F. Hong, T. H. Lee, Adaptive neural control of nonlinear time-delay systems with unknown virtual control coefficients, IEEE Trans. Syst. Man Cybern:B, 34 (2004), 499-516. 2.1

[4] S. S. Ge, J. Wang, Robust adaptive tracking for time-varying uncertain nonlinear systems with unknown control coefficients, IEEE Trans. Automat. Control, 48 (2003), 1463-1469. 2.2 
[5] Z.-G. Hou, A.-M. Zou, F.-X. Wu, L. Cheng, M. Tan, Adaptive dynamic surface control of a class of uncertain nonlinear systems in pure-feedback form using fuzzy backstepping approach, 4th IEEE Conference on Automation Science and Engineering, (2008), 821-826. 2

[6] C.-F. Hsu, C.-M. Lin, T.-T. Lee, Wavelet Adaptive Backstepping Control for a Class of Nonlinear Systems, IEEE Trans. Neural Netw., 17 (2006), 1175-1183. 1

[7] H. K. Khalil, Adaptive output feedback control of nonlinear systems represented by input-output models, IEEE Trans. Automat. Control, 41 (1996), 177-188. 1

[8] M. Krstic, I. Kanellakopoulos, P. Kokotovic, Nonlinear and Adaptive Control Design, Wiley, New York, (1995). 1

[9] P. Kumar, V. Panwar, D. Singh, An adaptive RBF controller for a class of non-affine nonlinear systems, Int. J. Math. Comput. Sci., 16 (2021), 67-77. 1

[10] F. L. Lewis, A. Yesildirek, K. Liu, Multilayer neural-net robot controller with guaranteed tracking performance, IEEE Trans. Neural Netw., 7 (1996), 388-399. 1

[11] T. Li, N. Pintus, G. Viglialoro, Properties of solutions to porous medium problems with different sources and boundary conditions, Z. Angew. Math. Phys., 70 (2019), 1-18. 1

[12] T. Li, G. Viglialoro, Analysis and explicit solvability of degenerate tensorial problems, Bound. Value Probl., 2018 (2018), 1-13. 1

[13] X. Li, D. Wang, Z. Peng, G. Sun, Adaptive fuzzy control for a class of non-affine systems, International Conference on Intelligent Control and Information Processing, (2010), 561-565. 1

[14] W. Lin, C. Qian, Adaptive control of nonlinearly parameterized systems: A nonsmooth feedback framework, IEEE Trans. Automat. Control, 47 (2002), 757-774. 1

[15] L. Liu, J. Huang, Global robust stabilization of cascade-connected systems with dynamic uncertainties without knowing the control direction, IEEE Trans. Automat. Control, 51 (2006), 1693-1699. 2.1, 3

[16] Z. Liu, G. Lai, Y. Zhang, X. Chen, C. L. P. Chen, Adaptive neural control for a class of nonlinear time-varying delay systems with unknown hysteresis, IEEE Trans. Neural Netw. Learn. Syst., 25 (2014), 2129-2140. 2.2

[17] W. Meng, Q. Yang, J. Si, Y. Sun, Adaptive Neural Control of a Class of Output-Constrained non-affine Systems, IEEE Trans. Cybernet., 46 (2016), 85-95. 1

[18] W. Meng, Q. Yang, Y. Ying, Y. Sun, Z. Yang, Y. Sun, Adaptive Power Capture Control of Variable-Speed Wind Energy Conversion Systems With Guaranteed Transient and Steady-State Performance, IEEE Trans. Energy Convers., 28 (2013), 716-725. 1

[19] V. Panwar, Wavelet neural network-based $\mathrm{H}^{\infty}$ trajectory tracking for robot manipulators using fast terminal sliding mode control, Robotica, 35 (2016), 1-16. 1, 2.1

[20] M. M. Polycarpou, Stable adaptive neural control scheme for nonlinear systems, IEEE Trans. Automat. Control, 41 (1996), 447-451. 1

[21] Z. Qu, Robust Control of Nonlinear Uncertain Systems, John Wiley \& Sons, New York, (1998). 1

[22] B. Ren, S. S. Ge, C.-Y. Su, T. H. Lee, Adaptive Neural Control for a Class of Uncertain Nonlinear Systems in Pure-Feedback Form With Hysteresis Input, IEEE Trans. Syst. Man Cybern: B, 39 (2009), 431-443. 2

[23] S. Sastry, M. Bodson, Adaptive Control: Stability, Convergence, and Robustness, Prentice-Hall, Upper Saddle River, NJ, (1989). 1

[24] J. J. Slotine, W. Li, Applied Nonlinear Control, Englewood Cliffs, NJ: Prentice hall, (1991). 1, 2

[25] V. I. Utkin, Sliding Modes in Control and Optimization, Springer-Verlag, Berlin, (1992). 1

[26] G. Viglialoro, T. E. Woolley, Boundedness in a parabolic-elliptic chemotaxis system with nonlinear diffusion and sensitivity and logistic source, Math. Methods Appl. Sci., 41 (2018), 1809-1824. 1

[27] D. Wang, J. Huang, W. Lan, X. Li, Neural network based robust adaptive control of nonlinear systems with unmodeled dynamics, Math. Comput. Simulation, 79 (2009), 1745-1753. 1

[28] A. Young, C. Cao, N. Hovakimyan, E. Lavretsky, An adaptive approach to non-affine control design for aircraft applications, AIAA Guidance, Navigation, and Control Conference and Exhibit, (2006), 1-28. 1

[29] J. Zhang, G. G. Walter, Y. Miao, W. N. W. Lee, Wavelet neural networks for function learning, IEEE Trans. Signal Process., 43 (1995), 1485-1497. 2.1 